\title{
In Vivo Reversible Regulation of Dendritic Patterning by Afferent Input in Bipolar Auditory Neurons
}

\author{
Yuan Wang and Edwin W Rubel \\ Virginia Merrill Bloedel Hearing Research Center, Department of Otolaryngology-Head and Neck Surgery, University of Washington School of Medicine, \\ Seattle, Washington 98195
}

\begin{abstract}
Afferent input regulates neuronal dendritic patterning locally and globally through distinct mechanisms. To begin to understand these mechanisms, we differentially manipulate afferent input in vivo and assess effects on dendritic patterning of individual neurons in chicken nucleus laminaris (NL). Dendrites of NL neurons segregate into dorsal and ventral domains, receiving excitatory input from the ipsilateral and contralateral ears, respectively, via nucleus magnocellularis (NM). Blocking action potentials from one ear, by either cochlea removal or temporary treatment with tetrodotoxin (TTX), leads to rapid and significant retraction of affected NL dendrites (dorsal ipsilaterally and ventral contralaterally) within $8 \mathrm{~h}$ compared with the other dendrites of the same neurons. The degree of retraction is comparable with that induced by direct deafferentation resulting from transection of NM axons. Importantly, when inner ear activity is allowed to recover from TTX treatments, retracted NL dendrites regrow to their normal length within $48 \mathrm{~h}$. The retraction and growth involve elimination of terminal branches and addition of new branches, respectively. Examination of changes in NL dendrites at $96 \mathrm{~h}$ after unilateral cochlea removal, a manipulation that induces cell loss in NM and persistent blockage of afferent excitatory action potentials, reveals a significant correlation between cell death in the ipsilateral NM and the degree of dendritic retraction in NL. These results demonstrate that presynaptic action potentials rapidly and reversibly regulate dendritic patterning of postsynaptic neurons in a compartment specific manner, whereas long-term dendritic maintenance may be regulated in a way that is correlated with the presence of silent presynaptic appositions.
\end{abstract}

\section{Introduction}

The branching patterns of dendritic arbors help shape firing patterns of a neuron and thus functional properties within neuronal circuits (Purves and Hume, 1981; Connors and Regehr, 1996; Yuste and Tank, 1996; Vetter et al., 2001; Schaefer et al., 2003). Both development and maintenance of dendritic arborization are regulated by afferent inputs (Cline, 2001; Wong and Ghosh, 2002; Redmond, 2008; Nagendran and Hardy, 2011). This regulation involves activity-dependent mechanisms mediated by neurotransmission (Sin et al., 2002; Lohmann and Wong, 2005; Redmond and Ghosh, 2005), as well as activityindependent mechanisms via diffusible cues or cell contact (Parrish et al., 2007; Urbanska et al., 2008). In vitro studies demonstrate that these mechanisms occur either globally to the whole neuron or locally to specific dendritic arbors or spines. How these distinct mechanisms work coordinately in a temporally and spatially controlled manner in dendritic regulation, however, is not well understood, especially in neurons under in vivo situations.

Received April 10, 2012; revised July 2, 2012; accepted July 7, 2012.

Author contributions: Y.W. and E.W.R. designed research; Y.W. performed research; Y.W. analyzed data; Y.W. and E.W.R. wrote the paper.

This work was supported by National Institute on Deafness and Other Communication Disorders Grants DC03829, DC-02739, DC-04661, and DC-00018 and a Deafness Research Foundation research grant.

Correspondence should be addressed to Edwin W. Rubel, Virginia Merrill Bloedel Hearing Research Center, Mail Stop 357923, University of Washington, Seattle, WA 98195. E-mail: rubel@uw.edu.

DOI:10.1523/JNEUROSCI.1737-12.2012

Copyright $\odot 2012$ the authors $\quad 0270-6474 / 12 / 3211495-10 \$ 15.00 / 0$
The chicken nucleus laminaris (NL) provides a useful model for studying afferent regulation of dendritic arborization. Dendrites of NL neurons segregate into dorsal and ventral domains, receiving excitatory input exclusively from the ipsilateral and contralateral ears, respectively, via the nucleus magnocellularis (NM) on each side of the brain (Parks and Rubel, 1975). Dendritic size and complexity are essentially symmetric between the two domains of the same NL neurons (Smith and Rubel, 1979; Smith, 1981; Deitch and Rubel, 1984), providing a fundamental cellular substrate for specialized function of NL in binaural hearing (Carr and Konishi, 1990). This symmetry is interrupted by transection of contralateral NM axons that induces retractions of ventral, but not dorsal, dendrites of the same NL neurons (Benes et al., 1977; Deitch and Rubel, 1984). This domain specificity allows in vivo studies of afferent influence on dendritic structure with a matched intracellular control. However, whether such observed dendritic retraction is an irreversible degeneration event or whether it represents a form of plasticity that reflects normal brain function remains unknown. In addition, specific mechanisms of afferent input that are responsible for the induced dendritic retraction have not been determined, although in vitro studies have demonstrated the importance of balanced afferent inputs and involvement of glutamate neurotransmission (Sorensen and Rubel, 2006, 2011).

The current study examined dendritic arborization and symmetry of individual NL neurons in vivo after a number of manipulations of their excitatory input from NM. These manipulations include direct axonal transection of contralateral NM projections 
and transneuronal blockage of presynaptic activity by unilateral cochlea removal or local tetrodotoxin (TTX) treatment to the inner ear. Each manipulation compromises excitatory input to one set of dendrites of NL neurons while leaving the input to the other set intact. All manipulations deprive action-potential-mediated neurotransmission to affected NL dendrites, whereas axonal transection also involves brain lesion and cochlea removal causes changes in presynaptic neurons in NM (Rubel and Fritzsch, 2002). Comparison of the effects of these manipulations on NL dendritic patterning helps identify key players in afferent regulation of dendritic structure.

\section{Materials and Methods}

White Leghorn chicks (Gallus gallus domesticus) of either sex were used 2-11 d after hatching. All procedures were approved by the University of Washington Institutional Animal Care and Use Committee and conformed to National Institutes of Health guidelines. All efforts were made to minimize pain and discomfort of the animals and to minimize the number of animals used.

\section{Manipulations of afferent inputs}

All procedures were described previously. Briefly, animals were anesthetized with a mixture of $40 \mathrm{mg} / \mathrm{kg}$ ketamine and $12 \mathrm{mg} / \mathrm{kg}$ xylazine. For transection of the crossed dorsal cochlear tract (XDCT), an ophthalmic knife was inserted through the fourth ventricle and into the brainstem to transect the XDCT at the midline (Deitch and Rubel, 1984; Wang et al., 2009). The XDCT contains the decussating NM axons that project to the ventral NL on both sides of the brain. For cochlea removal, the basilar papilla, including the lagena macula, was removed via the oval window after removal of the tympanic membrane and columella (Born and Rubel, 1985; Wang et al., 2009). The membranous cochlear duct was floated on water in a Petri dish and inspected under an operating microscope to ensure complete removal. For local TTX application in the inner ear, TTX (0.1 $\mu \mathrm{l}, 3 \mathrm{~mm}$; Sigma-Aldrich) was injected into the perilymph of the vestibular system at the saccule to avoid disrupting the integrity of the middle ear or cochlear fluids (Born and Rubel, 1988, Canady and Rubel, 1992). Each animal received either one injection or two injections $4 \mathrm{~h}$ apart. Control animals received injections of either distilled water or $0.9 \% \mathrm{NaCl}$. All vehicle or TTX treatments were applied to the left ear.

The auditory brainstem response (ABR) was tested at $0.5,4,8,24$, or $48 \mathrm{~h}$ after the first TTX application and was followed by transcardial perfusion of the animal. The contralateral (right) cochlea was removed immediately before the recording to limit the response to the manipulated (left) ear. The animal was exposed to tones at four frequencies $(0.5$, 1,2 , and $4 \mathrm{kHz}$ ) and various intensities (up to $90 \mathrm{~dB} \mathrm{SPL}$ ). The ABR was recorded using standard subcutaneous needle electrodes and averaged for a $20 \mathrm{~ms}$ window across 350 stimulus presentations. Tone bursts were $10 \mathrm{~ms}$ in duration with $2 \mathrm{~ms}$ rise-fall times. Threshold is defined as the lowest intensity stimulus to elicit a repeatable, visually discernible response of any averaged ABR wave within $10 \mathrm{~ms}$ of stimulus onset (Caras et al., 2010). For cases and stimulus frequencies that did produce a repeatable response at $90 \mathrm{~dB}$ SPL and below, the threshold was considered $100 \mathrm{~dB}$ SPL. Threshold was averaged for each frequency across all cases within the control or each experimental group.

\section{Cell filling in fixed sections}

Twenty animals were used for this experiment, including control animals $(n=4)$, those receiving transection of XDCT and surviving for $8 \mathrm{~h}(n=$ $3)$, those whose right cochlea was removed and survived for $8 \mathrm{~h}(n=3)$, and those treated with TTX and surviving for $8 \mathrm{~h}(n=6)$ or $2 \mathrm{~d}(n=4)$. After the manipulations and designated survival periods, the animals were deeply anesthetized and transcardially perfused with $0.9 \%$ saline, followed by $4 \%$ paraformaldehyde. The brains were removed from the skull, postfixed for $2-4 \mathrm{~h}$, and cut coronally at $50-100 \mu \mathrm{m}$.

A method of filling individual cells using electroporation in slice preparations (Sorensen and Rubel, 2006) was modified to efficiently fill individual NL cells in fixed sections. Briefly, sections were placed in a chamber mounted on the stage of a Leica fluorescent dissecting scope. A pulled glass pipette containing $5 \mathrm{~mm}$ Alexa Fluor 488 dextran (Invitrogen) was positioned within the line of NL cell bodies. A negative voltage $(20-60 \mathrm{~V}, 20 \mathrm{~ms}$ pulse duration, 20 pulses/s, $1-5 \mathrm{~s})$ was then applied to the section. This procedure results in labeling of the cell body, dendrites, and proximal axon of single NL cells (Fig. 1). To verify the completion of individual cell filling, sections were double labeled with immunoreactivity for microtubule-associated protein 2 (MAP2), which labels all neuronal cell bodies and dendrites in NL (Wang and Rubel, 2008). Monoclonal anti-MAP2 (catalog \#MAB3418) made in mouse was purchased from Millipore Bioscience Research Reagents. The antibody binds specifically to MAP2a and MAP2b and is detected as a $300 \mathrm{kDa}$ band in Western blot analysis.

\section{Cell reconstruction and dendritic analyses}

$3 D$ reconstruction. Complete dendritic arborizations of filled neurons were identified based on characteristic terminal morphology and the overlap with MAP2 immunoreactivity (Fig. 1). Only the neurons whose entire dendritic arborization was clearly labeled and confined within a single section were chosen for reconstruction. Image stacks were collected through the entire neuron with either $40 \times$ or $60 \times$ oil-immersion lens using an Olympus FV-1000 confocal microscope. These imaging settings provide sufficient resolution for accurate reconstruction and identification of terminal ending morphology. Image stacks were converted into a series of .tiff images in NIH ImageJ software (version 1.43X; National Institutes of Health) and then imported to Neurolucida (version 9.03; MBF Bioscience). The entire dendritic arborization was traced with lines through the middle of each branch. In some neurons, the thickness of the lines was adjusted to match the diameters of dendritic branches during reconstruction to verify the linear correlation between dendritic branch length and dendritic surface area reported previously (Deitch and Rubel, 1984). The majority of neurons were traced with lines of fixed thickness for efficiency.

Measurements. All measurements were made from 3D reconstructions of filled neurons using Neurolucida Explorer (version 9.03; MBF Bioscience). Total dendritic branch length (TDBL) was calculated as the sum of the length of all dendritic branches within the dorsal or ventral dendritic domain of a neuron. The total numbers of dendritic trees ( $\mathrm{N}$-trees), dendritic endings ( $\mathrm{N}$-endings), branch nodes ( $\mathrm{N}$-nodes), and branch segments (N-segs) were also counted for each dendritic domain. The 
average length of branch segments (L-segs) was calculated as TDBL divided by N-segs.

Analyses. Absolute TDBL values of individual NL neurons vary greatly and are associated with the physical location of the neuron along the tonotopic axis of the nucleus (Smith and Rubel, 1979). A previous study using Golgi-stained sections has reconstructed a large number of samples (592 neurons) before and after in vivo XDCT transection, grouped these neurons into eight sections along the tonotopic axis of the nucleus, and compared the absolute TDBL values of dorsal or ventral dendrites across neurons within the same tonotopic sections (Deitch and Rubel, 1984). These analyses revealed that, although the ventral (deprived) dendrites exhibit progressive retraction over time, the dorsal (intact) dendrites do not show significance changes in the absolute TDBL value within 2 weeks after the surgery. Compared with XDCT transection, cochlea removal and intracochlear TTX treatment are less invasive manipulations. Thus, we have every reason to assume that TDBL of the intact dendritic domains of the manipulated neurons remains unchanged within survival times examined (up to $4 \mathrm{~d}$ ). Thus, we used the intact dendritic domain as a reliable control to evaluate changes in deprived dendrites of the same neurons after manipulations used in the current study.

For each measured property, two types of analyses, dendritic polarity index (DPI) and percentage difference (PD), were conducted to quantify the effects of the manipulations on NL dendritic structure. Calculation of DPI and PD is described below using TDBL as an example but applies to all other measured properties.

DPI. DPI is an indicator of the polarity of the dendritic tree, independent of which, if either, dendritic domain is manipulated: DPI = $($ Dorsal - Ventral $) /($ Dorsal + Ventral $) \times 100$.

For example, the DPI of TDBL is the difference in TDBL between the two dendritic domains normalized to the sum of TDBLs of both domains (i.e., TDBL of the whole dendritic tree of a particular neuron). A DPI of zero indicates that the two domains have equal TDBLs. A positive DPI indicates a larger TDBL in the dorsal domain (TDBLd) than in the ventral domain (TDBLv). The opposite is true for a negative DPI.

$P D$. PD evaluates the degree of changes in dendritic structure of the manipulated domain compared with the other domain of the same neuron: $\mathrm{PD}=($ Manipulated - Control $) /$ Control $\times 100$.

In contrast to DPI, calculation of PD depends on which dendritic domain of a neuron is manipulated. For example, PD of TDBL after unilateral cochlea removal is the difference of TDBLv relative to the TDBLd for the neurons on the contralateral side and the difference of the TDBLd relative to the TDBLv for the neurons on the ipsilateral side. A PD of zero indicates balanced dendritic configuration, whereas negative and positive PDs indicate decreased and increased TDBL of the manipulated domain, respectively, compared with the control domain of the same neuron. For control unmanipulated neurons, "control" and "manipulated" domains were assigned to match the manipulated neurons being compared.

For statistic comparisons between control and experimental groups, each neuron is presented as a single data point. Comparisons using each animal as a single data point by pooling all reconstructed neurons from the same animal together yield similar results in terms of the mean, SEM, and $p$ values and will not be described further.

\section{Correlation analyses of $N L$ dendritic retraction with $N M$} cell death

Ten animals received cochlea removal from the right ear and survived for $4 \mathrm{~d}$. These animals and five unmanipulated animals were perfused as described above, and brain sections of $30 \mu \mathrm{m}$ thickness were immunostained for MAP2 using the peroxidase method (Wang and Rubel, 2008).

Quantification of dendritic retraction in NL. In this group of animals, all measurements and analyses were conducted in MAP2-stained sections from the left (contralateral) NL whose ventral dendrites were deprived. Because the percentages of dendritic retraction after afferent deprivation are comparable across the tonotopic axis of NL (Deitch and Rubel, 1984), one representative section was chosen from each case at the level of $50 \%$ caudal-to-rostral extent of NL. At this level, NL neurons have a characteristic frequency of $\sim 2 \mathrm{kHz}$ based on the tonotopic map of the nucleus (Rubel and Parks, 1975). Three or four photomicrographs were taken of
NL from the chosen section using a $63 \times$ objective attached to a Carl Zeiss microscope. For each photomicrograph, the dorsal and ventral dendritic domains occupied by MAP2-stained NL dendritic branches were outlined using NIH ImageJ software. The size of these dendritic areas in the intact domains ranged from $2-4 \times 10^{-3} \mathrm{~mm}^{2}$. Degree of dendritic retraction in NL was evaluated by $\mathrm{PD}$ of the ventral domain relative to the dorsal domain with respect to (1) the size of the dendritic areas and (2) the total MAP2 staining calculated as the dendritic area multiplying average optical density. In all cases, the comparison of the dendritic area and total MAP2 staining between the dorsal and ventral domains was made within the same photomicrograph from the same tissue section to avoid any possible inconsistencies caused by variations in immunostaining between cases and variations in brightness across photomicrographs. The PDs from a single animal were averaged across all images, and this mean PD represented a single data point for subsequent correlation analyses.

MAP2 staining provides a more efficient, although less sensitive, method to evaluate differences in dendritic length between the deprived and intact domains of a small group of NL neurons compared with analyses at the individual cell level. Although not sensitive enough to detect changes in dendritic length at early time points after the manipulations, MAP2 staining provides a reliable way to evaluate later changes when dendritic retraction accumulates and leads to substantial changes in the extension of the dendritic filed at several days after afferent deprivation. This analysis has been used to study dynamics of a number of cellular events after the same manipulations (Durham and Rubel, 1985; Wang and Rubel, 2008; Wang et al., 2009).

Quantification of cell death in NM. The amount of neuronal death in NM attributable to removal of the ipsilateral cochlea (Born and Rubel, 1985) was evaluated by PDs in neuron number of the right, deprived NM relative to the left, intact NM. For each case, neurons were counted from an MAP2-stained section containing NM neurons with a characterized frequency of $2 \mathrm{kHz}$, which is approximately at the $60 \%$ caudal-to-rostral extent of NM (Rubel and Parks, 1975). Labeled neurons were counted using a $40 \times$ objective and eyepiece reticule. Neurons without an obvious nuclear profile were excluded from counting. Abercrombie's formula was used to correct cell profile counting (Abercrombie, 1946): $N=T /(T+D) \times$ $n$, where $N$ is number of cells, $T$ is section thickness, $D$ is mean diameter of 12 nuclei, and $n$ is number of counted nuclei.

PD in cell number was calculated for each case and plotted as a function of PD in dendritic retraction of NL neurons.

\section{Statistics}

Significance was determined by two-tailed $t$ tests and correlation analyses, linear regression, one-way ANOVA followed by Bonferroni's multiple comparison test, or two-way ANOVA followed by Bonferroni's post hoc test, using the Prism version 5 software package (GraphPad Software). $p<0.05$ was considered statistically significant. All data are shown as mean \pm 1 SEM in the text and figures.

\section{Results}

\section{Activity deprivation leads to rapid dendritic retraction of NL neurons}

Previous studies reported that transection of the XDCT, which contains NM axons projecting to ventral dendrites of NL neurons on both sides of the brain, leads to rapid retraction of ventral dendrites (Benes et al., 1977; Deitch and Rubel, 1984). To examine whether induced alterations in neuronal activity are responsible for the retraction, we manipulated afferent inputs to NL dendrites transneuronally by unilateral cochlea removal in vivo (Fig. 2A,B). Cochlea removal eliminates action potentials to the ipsilateral NM and then evoked glutamatergic signaling to the dorsal dendrites of the ipsilateral NL and ventral dendrites of the contralateral NL without directly damaging NM axons (Born et al., 1991). Somatodendritic morphology of individual NL neurons was visualized by dye filling at $8 \mathrm{~h}$ after this surgery. TDBL was measured separately for TDBLd and TDBLv from 3D recon- 
A

ear

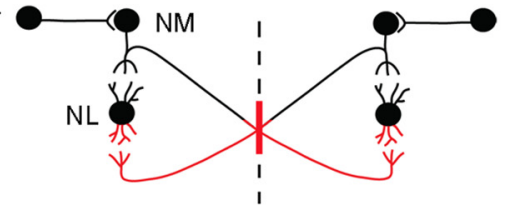

B

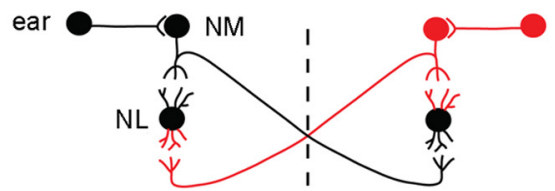

C

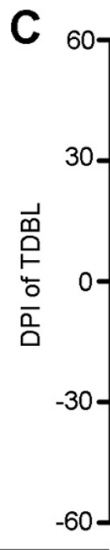

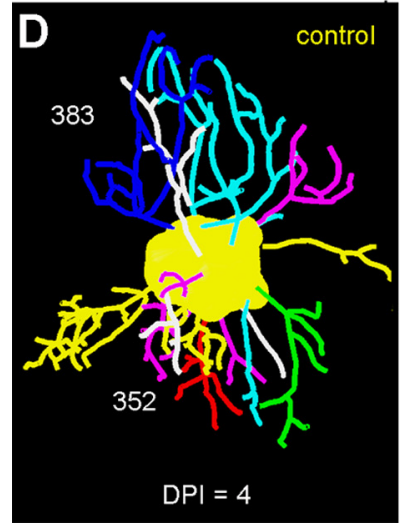
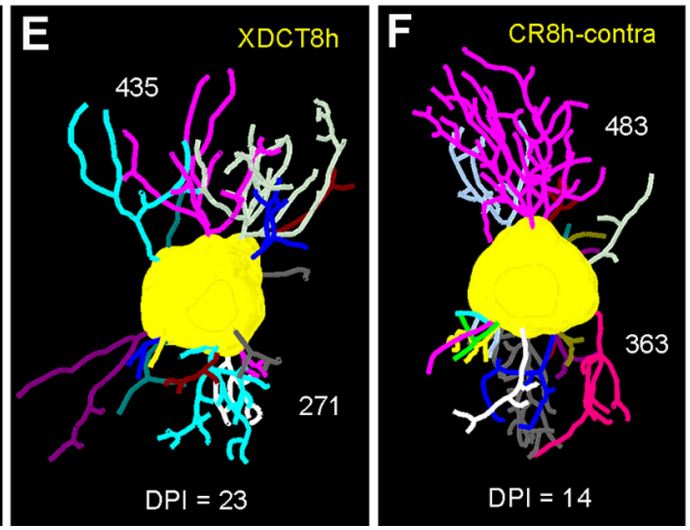

$\star \star$
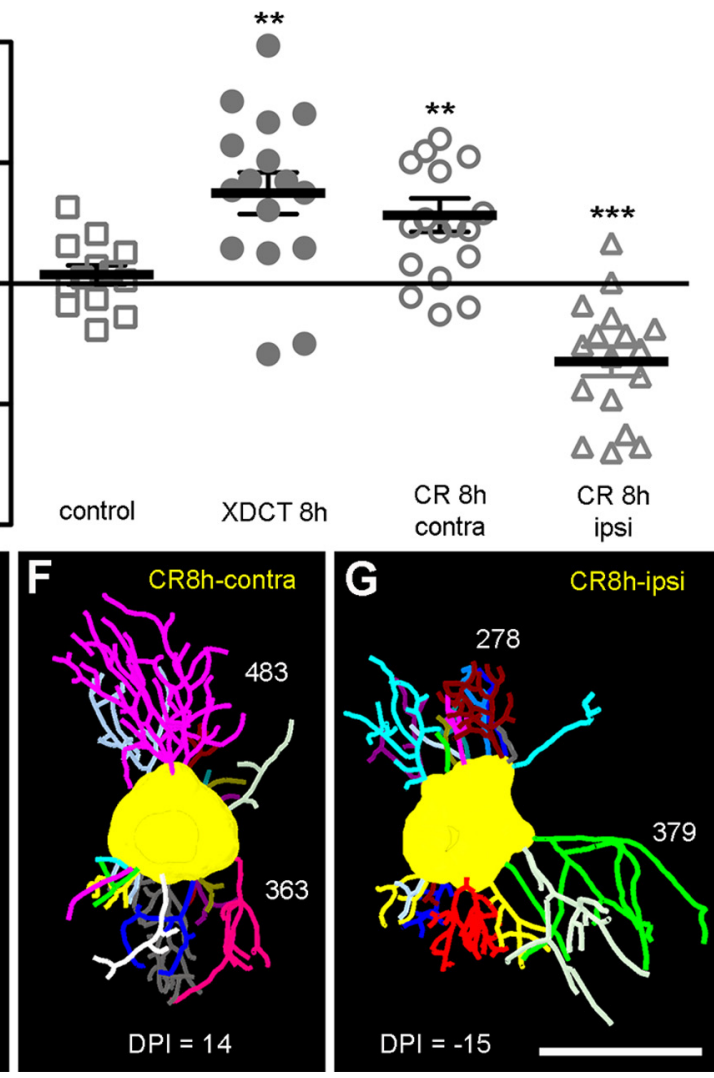

Figure 2. Effects of transection of the XDCT and unilateral cochlea removal (CR) on TDBL of NL neurons. $A, B$, Schematic drawings illustrate the surgical site for each manipulation and deprived axons and dendrites (red) influenced by each manipulation. Dashed lines indicate the midline. C, DPI of TDBL of individual NL neurons is plotted as a function of the manipulation at $8 \mathrm{~h}$. Neurons whose ventral dendrites are deprived (XDCT $8 \mathrm{~h}$ and CR $8 \mathrm{~h}$ contra) exhibit significantly larger DPIs compared with control neurons, indicating significantly smaller TDBL in the ventral domain. The opposite is true for the neurons with deprived dorsal dendrites (CR $8 \mathrm{~h}$ ipsi). Each data point (gray) represents an individual neuron. Mean and SEM are indicated by black bars for each group. The asterisks above individual groups indicate that the DPI of a manipulation group is significantly different $\left({ }^{* *} p<0.001\right.$; $\left.{ }^{* * *} p<0.0001\right)$ from the control group. $D-G, 3 D$ reconstructions of representative filled neurons in control (D) and after XDCT transection $(\boldsymbol{E})$ and contralateral $(\boldsymbol{F})$ and ipsilateral $(\boldsymbol{G})$ cochlea removal. TDBL (micrometers) of each dendritic domain and DPI are indicated in the tracing for each neuron. Dorsal is up and ventral is down. Scale bar: (in G) D-G, $20 \mu \mathrm{m}$. contra, Contralateral; ips, ipsilateral.

structions of NL neurons. DPI of TDBL was calculated as $($ TDBLd - TDBLv $) /($ TDBLd + TDBLv $) \times 100$ for each neuron. Positive and negative DPIs indicate longer dendritic length dorsally and ventrally, respectively.

For control neurons ( $n=13$ neurons from 4 animals), TDBLd and TDBLv are significantly correlated $\left(r^{2}=0.45, p=0.01\right)$. The average TDBLd is slightly larger than TDBLv, with the average DPI slightly positive ( $2.2 \pm 2.4$; Fig. $2 C, D)$, although this difference is not statistically significant. Consistent with previous reports, XDCT transection led to rapid retraction of the ventral dendrites (Figs. 2E, 3A). Within $8 \mathrm{~h}$, the TDBLv was significantly smaller than TDBLd ( $n=16$ neurons from 3 animals, $p=$ $0.0004)$, with the DPI $(22.5 \pm 5.2)$ significantly larger than the control $(p=0.003$; Fig. $2 C)$.

After unilateral cochlea removal, neurons on the side contralateral to the surgery exhibited retraction of their ventral dendrites similar to that seen after XDCT transection (Figs. 2C,F, $3 A$ ). Within $8 \mathrm{~h}$, the TDBLv was significantly smaller than TDBLd $(n=18$ neurons from 3 animals, $p=0.0005)$, with the DPI $(17.0 \pm 4.2)$ significantly larger than the control $(p=0.01)$ but not significantly different from the neurons receiving XDCT transection. On the side ipsilateral to the surgery, the dorsal dendrites underwent similar retractions (Figs. $2 C, G, 3 A$ ). Within $8 \mathrm{~h}$, the TDBLd was significantly smaller than TDBLv $(n=17, p<$ $0.0001)$, with the DPI $(-19.4 \pm 3.7)$ significantly smaller than the control $(p<0.0001)$. One-way ANOVA analysis with multiple comparisons further demonstrated significant and differential effects of unilateral cochlea removal on dendritic polarity of NL neurons $(p<0.0001)$. As a more direct representation of the degree of changes in TDBL, PDs of the manipulated domain relative to the other domain of the same neuron are listed in Table 1. These data demonstrate that deprivation of afferent action potentials leads to rapid reduction in dendritic length of NL neurons in a compartment-specific manner.

\section{Dendritic retraction induced by activity deprivation} is reversible

To understand the role of activity in this afferent regulation, it is important to clarify whether dendritic retraction induced by activity deprivation is dynamically regulated or whether it represents irreversible pathological events. We thus examined whether dendritic retractions are reversible when afferent activity is resumed. TTX, a sodium channel blocker, was delivered to the inner ear to temporarily block the production of action potentials from the cochlea to the brainstem (Fig. 4A). Similar to cochlea removal, TTX delivery eliminates action potential-dependent signaling to the ipsilateral NM and then to the dorsal dendrites of the ipsilateral NL and ventral dendrites of the contralateral NL (Born and Rubel, 1988; Born et al., 1991). To maintain this blockage for at least $8 \mathrm{~h}$, the same time point examined after XDCT transection or unilateral cochlea removal, each animal was given two deliveries of TTX $4 \mathrm{~h}$ apart (Fig. 4A). Time course and dura- 

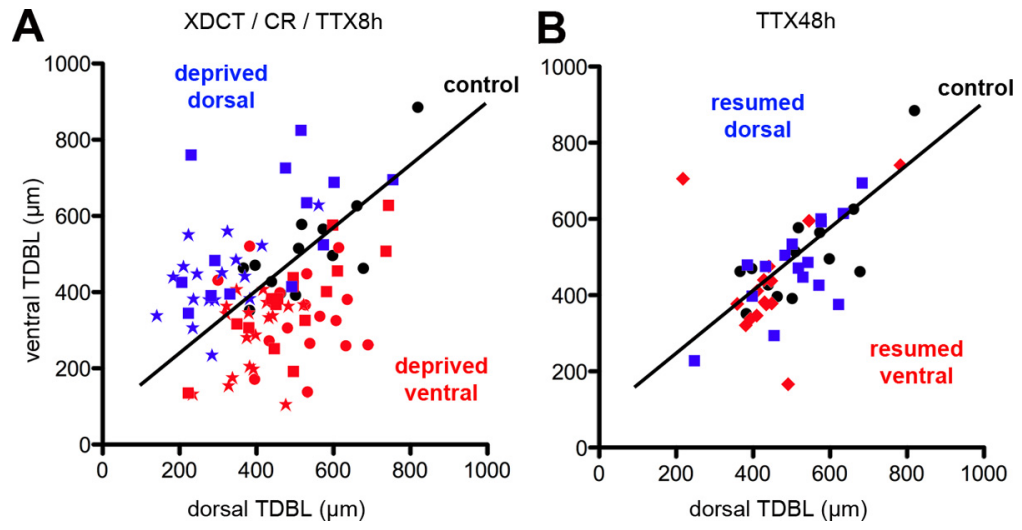

Figure 3. Absolute TDBL values of analyzed neurons after manipulations. $A$, TDBL values of neurons after XDCT transection (red circles), unilateral cochlea removal (red and blue stars), and TTX treatment (red and blue squares) at $8 \mathrm{~h}$ compared with control neurons (black circles). $\boldsymbol{B}$, TDBL values of neurons after TTX treatment (red and blue squares) at $48 \mathrm{~h}$ compared with control neurons (black circles). For both $\boldsymbol{A}$ and $\boldsymbol{B}$, each data point represents an individual neuron. Neurons with manipulated dorsal and ventral dendrites are in red and blue, respectively. Solid black lines are linear regression of control neurons. Note that the majority of filled neurons are located in the rostral and middle portions of the nucleus and have a TDBL of 150-900 $\mu \mathrm{m}$ in one domain. One neuron filled on the ipsilateral side at $8 \mathrm{~h}$ after TTX treatment has TDBLd and TDBLv of 1162 and $1244 \mu \mathrm{m}$, respectively, and is not included in the graph. with control neurons, the DPI of TTXtreated neurons that survived $48 \mathrm{~h}$ was not significantly different in terms of TDBL (ipsilateral, $3.7 \pm 2.4, n=16$ neurons from 4 animals; contralateral, $2.9 \pm 1.3$, $n=15$ neurons). The results of two-way ANOVA for the survival time after TTX treatment ( 8 vs $48 \mathrm{~h}$ ) and the domain of the deprived dendrites (dorsal vs ventral) indicated that the degree of changes in TDBL of manipulated dendrites was determined by the survival time after TTX treatment, i.e., the level of activity, and this effect did not depend on whether the manipulated domain was dorsal or ventral. These data indicate that retracted NL dendrites induced by activity deprivation regrew to their original length after their normal afferent activity was resumed, demonstrating dynamic regulation of afferent action potentials on dendritic length of NL neurons. tion of the effect of TTX treatments on NM/NL activity were evaluated by ABR (Fig. 4B,D). Within $30 \mathrm{~min}$ of the first TTX delivery (Fig. $4 B$, red line), there was a $40 \mathrm{~dB}$ or greater increase in $\mathrm{ABR}$ threshold in response to pure tones. Because the maximum output of the sound delivery system used in this study was $90 \mathrm{~dB}$, we cannot say that all responses were eliminated, but the changes were similar to those in our previous studies (Born and Rubel, 1988; Born et al., 1991). These increases lasted for at least $4 \mathrm{~h}$ for one TTX delivery (blue line) and $8 \mathrm{~h}$ for two deliveries (green line). ABR threshold returned to the pretreatment level within $24 \mathrm{~h}$ (gray line), indicating that this treatment did not result in permanent damage to the inner ear, or eighth-nerve cell bodies or axons or cells in the cochlear nuclei.

TTX blockage of eighth-nerve activity produced a pattern of dendritic reorganization in NL that was indistinguishable from unilateral cochlear removal within $8 \mathrm{~h}$; dorsal dendrites of the ipsilateral NL and ventral dendrites of the contralateral NL exhibited significantly smaller TDBL compared with the other domain of the same neurons (ipsilateral, $p=0.008$; contralateral, $p<0.0001$; Figs. $3 A, 4 C, E, F)$. Compared with the control neurons, DPI of TDBL of TTX-treated NL neurons was significantly smaller on the ipsilateral side $(-14.7 \pm 4.5, n=14$ neurons from 6 animals, $p=0.003$ ) and significantly larger on the contralateral side $(14.8 \pm 2.7, n=16$ neurons, $p=0.002)$. This significance was confirmed by one-way ANOVA with multiple comparisons $(p<0.0001)$. A two-way ANOVA of DPI (manipulation by side) did not yield significant interaction between these two parameters, indicating that the effect of deprivation on dendritic polarity of TDBL within $8 \mathrm{~h}$ was determined by which dendritic domain was deprived but did not depend on whether the deprivation was induced by TTX treatment or cochlea removal. These data further demonstrate that action-potential-dependent signaling comprises a major component of the afferent activity responsible for rapid structural regulation of NL dendrites.

Because ABR threshold returned to the control level within $24 \mathrm{~h}$ after TTX treatment, we examined the dendritic organization of NL neurons at $48 \mathrm{~h}$ after surgery, so that these neurons had at least $24 \mathrm{~h}$ experience with resumed afferent activity (Fig. 4A). On either side of the brain, TDBLd and TDBLv were not significantly different from each other (Figs. $3 B, 4 C$ ). When compared

\section{Afferent activity regulates dendritic complexity of NL neurons}

To explore cellular compartments underlying activity regulation of dendritic structure, we measured a number of properties related to dendritic structural complexity and examined correlations of changes in these properties with changes in TDBL. DPI of each property was calculated for each neuron as described for TDBL. To simplify the description, neurons from animals that received XDCT transection, unilateral cochlea removal, or TTX treatment and survived for $8 \mathrm{~h}$ are grouped into two categories based on the domain of the deprived dendrites.

The most distinct changes after afferent deprivation, along with decreases in TDBL, are decreases in N-endings, $\mathrm{N}$-nodes, and $\mathrm{N}$-segs (Fig. $5 A-C)$. In control neurons $(n=13)$, ventral dendrites on average have $\sim 10 \%$ more endings, nodes, and segments than the dorsal dendrites of the same neurons, although only the difference in $\mathrm{N}$-segs is statistically significant $(p=$ 0.026). After differential deprivation, NL neurons exhibited significantly smaller $\mathrm{N}$-endings, $\mathrm{N}$-nodes, and $\mathrm{N}$-segs in the deprived domain compared with the other domain (for all, $p<$ $0.0001)$. Compared with the control neurons, DPIs of N-endings, $\mathrm{N}$-nodes, and $\mathrm{N}$-segs were significantly smaller in the neurons with deprived dorsal dendrites $(p=0.004-0.007, n=31)$ and significantly larger in the neurons with deprived ventral dendrites $(p=0.0004-0.002, n=50)$. This pattern of changes was confirmed by one-way ANOVA with multiple comparisons $(p<$ $0.0001)$. On average, $\mathrm{N}$-endings, $\mathrm{N}$-nodes, and $\mathrm{N}$-segs of the deprived domain were $\sim 30 \%$ smaller for deprived dorsal domain and $\sim 20 \%$ smaller for deprived ventral domain than the other domain of the same neuron (Table 1). Importantly, restoring activity caused the $\mathrm{N}$-endings, $\mathrm{N}$-nodes, and $\mathrm{N}$-segs to return to the control levels. In contrast, L-segs, calculated as TDBL divided by N-segs, remained unchanged after manipulations (Fig. 5D), although the ventral domain had a significantly smaller L-seg than the dorsal domain in the control and some manipulated groups (Table 1). In addition, neurons under these three conditions show similar distribution of segment length between the dorsal and ventral domains. No significant difference in the variation of L-segs was detected between the control and manipulated groups (Fig. 5E). 
Table 1. PD in structural properties of NL neurons after afferent manipulations

\begin{tabular}{|c|c|c|c|c|c|c|c|}
\hline & $n$ & TDBL & $\mathrm{N}$-trees & $\mathrm{N}$-endings & N-nodes & $\mathrm{N}$-segs & L-segs \\
\hline \multirow[t]{2}{*}{ Control } & 13 & $-3.0 \pm 4.5$ & $2.1 \pm 13.6$ & $13.0 \pm 8.0$ & $10.6 \pm 8.8$ & $17.3 \pm 5.5$ & $-14.6 \pm 2.9$ \\
\hline & & $7.5 \pm 5.7$ & $42.4 \pm 39.3$ & $-1.0 \pm 13.1$ & $4.6 \pm 13.1$ & $-11.8 \pm 5.3$ & $18.8 \pm 4.3$ \\
\hline Deprived ventral & 50 & $-27.1 \pm 3.5$ & $4.4 \pm 7.5$ & $-19.6 \pm 3.8$ & $-24.0 \pm 4.5$ & $-16.8 \pm 4.4$ & $-8.2 \pm 2.8$ \\
\hline XDCT $8 \mathrm{~h}$ & 16 & $-31.6 \pm 8.1$ & $-4.9 \pm 10.5$ & $-19.3 \pm 10.0$ & $-23.1 \pm 11.0$ & $-14.7 \pm 11.5$ & $-10.1 \pm 3.8$ \\
\hline CR 8 h contra & 18 & $-25.5 \pm 5.9$ & $13.7 \pm 13.0$ & $-22.9 \pm 2.6$ & $-29.6 \pm 5.1$ & $-25.8 \pm 4.1$ & $1.3 \pm 4.7$ \\
\hline TTX 8 h contra & 16 & $-24.4 \pm 3.8$ & $5.6 \pm 14.4$ & $-17.0 \pm 3.6$ & $-20.0 \pm 5.1$ & $-10.8 \pm 4.2$ & $-14.6 \pm 3.6$ \\
\hline \multicolumn{8}{|l|}{ Resumed ventral } \\
\hline TTX 48 h contra & 15 & $-5.2 \pm 2.5$ & $5.1 \pm 8.3$ & $3.7 \pm 5.8$ & $1.7 \pm 5.8$ & $2.3 \pm 5.8$ & $-4.2 \pm 4.5$ \\
\hline Deprived dorsal & 31 & $-26.4 \pm 4.2$ & $-11.5 \pm 8.3$ & $-31.2 \pm 3.9$ & $-31.4 \pm 5.0$ & $-31.6 \pm 4.0$ & $10.4 \pm 5.1$ \\
\hline CR $8 \mathrm{~h}$ ipsi & 17 & $-29.8 \pm 5.4$ & $-23.3 \pm 6.5$ & $-29.1 \pm 4.2$ & $-28.7 \pm 4.5$ & $28.9 \pm 4.1$ & $-1.1 \pm 6.0$ \\
\hline TTX 8 h ipsi & 14 & $-22.3 \pm 6.6$ & $3.3 \pm 12.2$ & $-33.1 \pm 5.7$ & $-33.7 \pm 8.0$ & $-33.9 \pm 5.9$ & $20.3 \pm 6.0$ \\
\hline \multicolumn{8}{|l|}{ Resumed dorsal } \\
\hline TTX 48 h ipsi & 16 & $9.8 \pm 5.8$ & $20.0 \pm 15.6$ & $2.6 \pm 8.9$ & $3.3 \pm 9.3$ & $3.3 \pm 9.2$ & $12.7 \pm 6.6$ \\
\hline
\end{tabular}

The second column $(n)$ indicates the number of neurons analyzed in each group. PD is calculated as the percentage difference of the manipulated domain relative to the other domain for manipulated groups. For control neurons, PD is the percentage difference of the ventral domain relative to the dorsal domain when compared with groups with manipulated ventral domain (first row) and of the dorsal domain relative to the ventral domain when compared with groups with manipulated dorsal domain (second row). Data are present as mean \pm SEM.

In addition, we analyzed the correlation between TDBL and $\mathrm{N}$-endings separately for the dorsal and ventral domains under control and manipulated conditions. Linear regressions of the correlation showed a significant trend of increasing TDBL with larger $\mathrm{N}$-endings $(p<0.0001)$. Importantly, PD of TDBL induced by manipulations significantly correlated with the PD of N-endings $(p<0.0001$; Fig. $5 F)$. Neurons with larger PDs in TDBL tend to have larger PDs in N-endings. These data indicate that changes in $\mathrm{N}$-endings, i.e., terminal branches, reflect changes in TDBL.

Conversely, the morphology of dendritic endings is not affected by the manipulations. In control neurons, $>80 \%$ of dendritic terminal endings are characterized with an enlarged bulge with (type 1; Fig. $1 B$, green arrowheads) or without (type 2; red arrows) narrow extensions. Terminals without a detectable enlarged bulge are grouped as type 3 endings (yellow arrowheads). After the manipulations, these three types of terminal endings were still present in both dendritic domains of each neuron. In addition, the percentage of each ending type in the deprived domain, as quantified from neurons whose ventral dendrites were deprived by XDCT transection, remained unchanged compared with the control domain. It is also worth noting that the $\mathrm{N}$-tree in each domain was not significantly altered by manipulations of afferent inputs, consistent with a previous report (Deitch and Rubel, 1984).

\section{Dendritic maintenance of NL neurons is correlated with cell survival of NM neurons}

To explore whether the integrity of presynaptic contacts regulate NL dendritic structure in vivo in the absence of action potentials, we took advantage of the observation that unilateral cochlea removal induces cell death in NM within $2 \mathrm{~d}$ (Born and Rubel, 1985). The percentage of NM neurons that die varies greatly among the individual cases. This variation will presumably affect the pattern and/or the total number of presynaptic terminals apposed to NL dendrites. The association of dendritic maintenance in NL with the cell survival of their presynaptic NM neurons was estimated by examining the correlation between the degree of dendritic retraction in NL and the percentage of cell death in NM after unilateral cochlea removal. MAP2 immunocytochemistry was used as a somatodendritic marker for dendritic measurements in NL and cell counting in NM.

In control animals, the average PD in cell number between the left and right $\mathrm{NM}$ is close to zero $(-2.3 \pm 9.7, n=5$ animals), indicating that cell numbers in the two NMs are nearly equivalent. In NL, the size of the dendritic area occupied by MAP2 staining is slightly smaller in the ventral domain than in the dorsal domain with a relative PD of $-13.8 \pm 14.3$ (Fig. $6 I$, white circle). Consistent with previous studies, each animal that survived $4 \mathrm{~d}$ after unilateral cochlea removal ( $n=10$ animals) had fewer neurons in the ipsilateral NM than the contralateral NM. PDs in cell number of the ipsilateral NM relative to the contralateral NM varied greatly, ranging from -8.8 to -71.9 (Fig. 6). Within $\mathrm{NL}$, the ventral dendrites of the contralateral side and the dorsal dendrites of the ipsilateral side exhibited retraction compared with the other, non-deprived, domain of the same neurons. PDs in the dendritic area of the ventral domain relative to the dorsal domain also varied greatly, ranging from -19.5 to -67.3 , as calculated from the contralateral NL. Importantly, more dramatic dendritic retractions were detected in cases with greater cell loss in the deprived NM ipsilateral (Fig. 6A-- $H$ ). Linear trend analysis revealed a highly significant trend toward increasing changes in NL dendritic area as a function of changes in NM cell number $\left(r^{2}=\right.$ $0.58, p<0.0001$; Fig. $6 I$, black circles and line).

Because this manipulation may lead to decreases in the density of dendritic branches in the deprived domain in addition to a reduction in dendritic area at $4 \mathrm{~d}$, changes in NL dendritic structure were also evaluated by PDs of total MAP2 staining, calculated as the dendritic area multiplied by the average density of MAP2 staining. Importantly, PD in total MAP2 staining in NL correlated with PD in cell number of NM in a similar manner as PD in dendritic area $\left(r^{2}=0.48, p<0.0001\right.$; Fig. $6 I$, gray triangles and line).

\section{Discussion}

One important observation of the current study is that afferentdeprivation-induced early changes in NL dendritic structure is reversible with the resumption of hearing and presynaptic excitatory action potentials. This reversibility demonstrates that retracting NL dendrites, after afferent deprivation, maintain a high degree of flexibility and actively respond to changes in afferent input in either direction. Although afferent or sensory deprivation has been shown to slow dendritic growth or affect dendritic stability in a number of systems (Groc et al., 2002; Wong and Ghosh, 2002; Mizrahi and Katz, 2003), the current study provides the first in vivo observation that such changes in dendritic branch length can be reversed by recovery of normal afferent activity. Dynamic plasticity in the auditory brainstem has also been dem- 

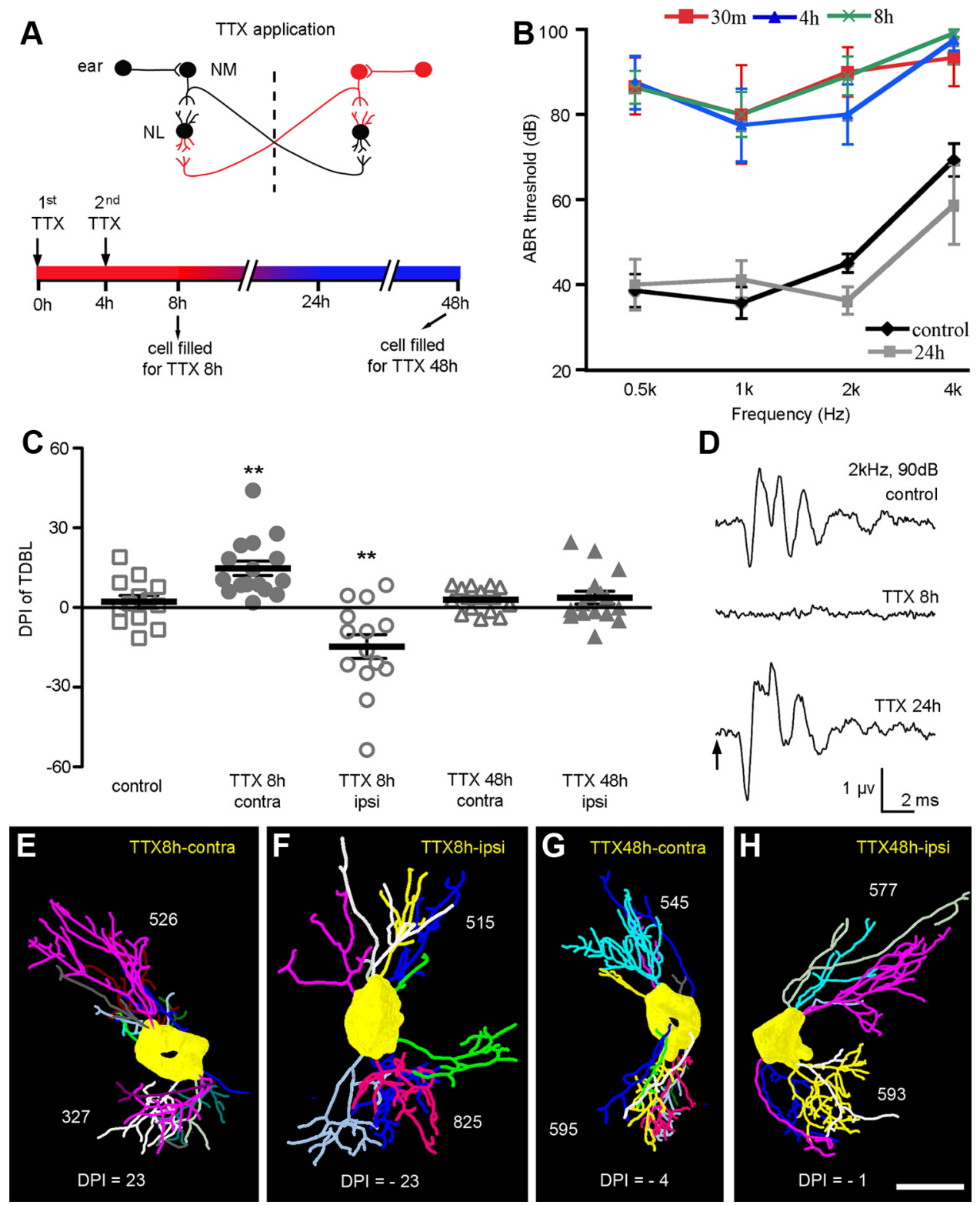

D
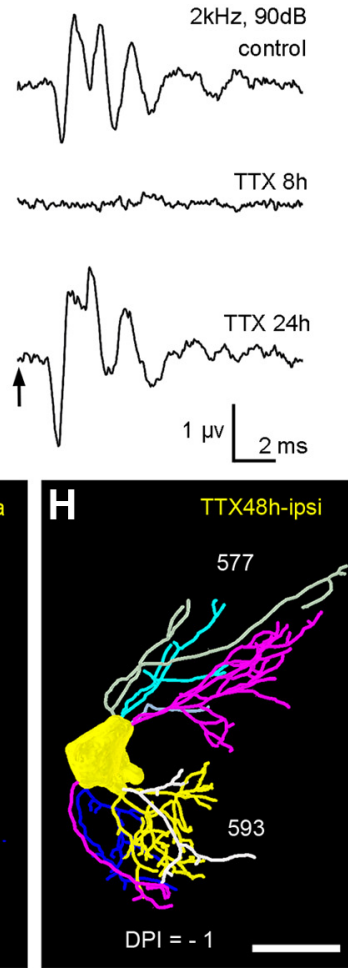

Figure 4. Effects and time course of local $T$ TX treatment on ABR and TDBL of NL neurons. $A$, Schematic drawings illustrate affected axonal and dendritic fields (red) after $T$ TX treatment (top) and time course of TTX treatment and cell filling (bottom). Level of ABR activity is indicated along the time course with red and blue colors coding reduced or normal levels of activity, respectively. $\boldsymbol{B}$, ABR threshold is plotted as a function of the frequency of sound stimulation. ABR thresholds increase dramatically at all examined frequencies at $30 \mathrm{~min}$ (red), $4 \mathrm{~h}$ (blue), and $8 \mathrm{~h}$ (green) and return to the control levels (black) by $24 \mathrm{~h}$ (gray). C, DPI of TDBL of individual NL neurons at 8 and $48 \mathrm{~h}$ after TTX treatment. Lack of afferent activity produces similar degrees and patterns of dendritic retraction in NL as cochlea removal during the first $8 \mathrm{~h}$. These changes are reversed after $48 \mathrm{~h}$ on both sides of the brain. Each data point (gray) represents an individual neuron. Mean and SEM are indicated by black bars for each group. The asterisks above individual groups indicate that the DPI of a survival group is significantly different $\left.{ }^{* *} p<0.001\right)$ from the control. $D$, Examples of ABR waveforms in response to $90 \mathrm{~dB}$ pure tone stimulation at $2 \mathrm{kHz}$ in control, 8 and $24 \mathrm{~h}$ after $T T X$ treatment. Arrow indicates the onset of stimulation. $\boldsymbol{E}-\boldsymbol{H}, 3 \mathrm{D}$ reconstructions of representative filled neurons on the contralateral and ipsilateral sides at $8 \mathrm{~h}(\boldsymbol{E}, \boldsymbol{F})$ and $48 \mathrm{~h}(\boldsymbol{G}, \boldsymbol{H})$ after $T$ TXX treatment. TDBL (micrometers) of each dendritic domain and DPI are indicated in the tracing for each neuron. Dorsal is up and ventral is down. Scale bar: (in $\boldsymbol{H}) \boldsymbol{E}-\boldsymbol{H}, 20 \mu \mathrm{m}$. contra, Contralateral; ipsi, ipsilateral.

onstrated recently in deaf cats in which electrical stimulation through cochlear implants has a restorative effect on synaptic organization in the medial superior olive, the mammalian analogs of the avian NL (Tirko and Ryugo, 2012). These forms of in vivo plasticity are consistent with studies demonstrating that enriched sensory inputs or electrical stimulation enhances dendritic growth (Sin et al., 2002; Faherty et al., 2003).

Similar to the rapidity of the retraction, NL dendrites appear to regrow quickly with a full recovery of their branch length in $24-36 \mathrm{~h}$ after $20-30 \%$ retraction. It is interesting to note that this high dynamics may be manifested in the unique terminal morphology of NL dendrites. Dendritic endings and some branch points of NL neurons possess an enlarged bulb with or without narrow extensions, morphologically resembling growth cones and filopodia of developing dendrites (Mattila and Lappalainen, 2008; Heiman and Shaham, 2010). An ending may quickly turn into a branch point by elongating one of its filopodial-like extensions into a branch. Similarly, a branch point becomes an ending if its daughter branches retract into narrow extensions or completely disappear. This presumably fast switch between endings and branch points may provide a fundamental cellular substrate for fast dendritic reorganization in NL neurons.

The similarity in the degree of dendritic retraction at $8 \mathrm{~h}$ after direct axonal transection and transneuronal deprivation of action potentials achieved by removal of the cochlea or injection of TTX into the cochlea indicates that these initial retractions pri- 
marily result from acute elimination of excitatory presynaptic action potentials. This observation is seemingly in conflict with reports that signaling mediated by action potentials is not required for dendritic development and maintenance in general (Rajan and Cline, 1998; Rajan et al., 1999; Wong et al., 2000) and that miniature spontaneous presynaptic neurotransmitter release is sufficient for maintaining NL dendritic structure in vitro (Sorensen and Rubel, 2006). However, other reports suggest that the pattern or competition between the inputs, rather than the absolute level of activity, is important for dendritic (including NL) and axonal maintenance (Katz and Constantine-Paton, 1988; Kossel et al., 1995; Kleim et al., 1997; Hata et al., 1999; Tailby and Metha, 2004; Hua et al., 2005; Hashimoto et al., 2009; Sorensen and Rubel, 2006; 2011). It is known that NM neurons normally spike at a very high frequency, even in the absence of acoustic stimulation (Stopp and Whitfield, 1961; Warchol and Dallos, 1990; Born et al., 1991). The balance between the two sets of excitatory inputs to NL is primarily determined by differences in the spike rate of the ipsilateral and contralateral NM axons. This high rate of spiking in NM is not maintained after cochlea removal, perilymphatic TTX exposure, or in isolated brain slice preparations, because it is entirely derived from the activity of inner ear hair cells (Born et al., 1991; Lippe, 1994). In the absence of action potentials, minis probably become the major player in determining the balance between inputs and probably dendritic maintenance.

Dendritic complexity analyses of NL neurons demonstrate that early changes in TDBL are accompanied by changes in the number of endings in the same directions, suggesting that elimination of a number of terminal arbors contributes to reductions in TDBL after afferent deprivation. This observation is consistent with in vitro time-lapse imaging showing that XDCT transectioninduced dendritic retraction takes place primarily at the distal portion of the neuron (Sorensen and Rubel, 2006). Similarly, increases in TDBL after afferent restoration involve addition of new branches, although it may also involve growth of existing branches. In addition, our correlation analyses have revealed that changes in the number of dendritic endings can be used as a reliable indicator of changes in TDBL, suggesting that these eliminations and additions of terminal arbors contribute substantially to changes in TDBL. Furthermore, in vitro time-lapse imaging has demonstrated that XDCT transection results in retraction of approximately half of the dendritic branches, whereas the other half remain stable or are growing (Sorensen and Rubel, 2006). These observations suggest that changes in TDBL after afferent manipulations do not take place uniformly along all affected dendritic branches and may occur in a branch-specific manner by which the fate (growing, retracting, or remaining stable) of individual branches is influenced by a combination of afferent inputs and intrinsic properties of each branch. This suggestion is consisto have larger PDs in N-endings.
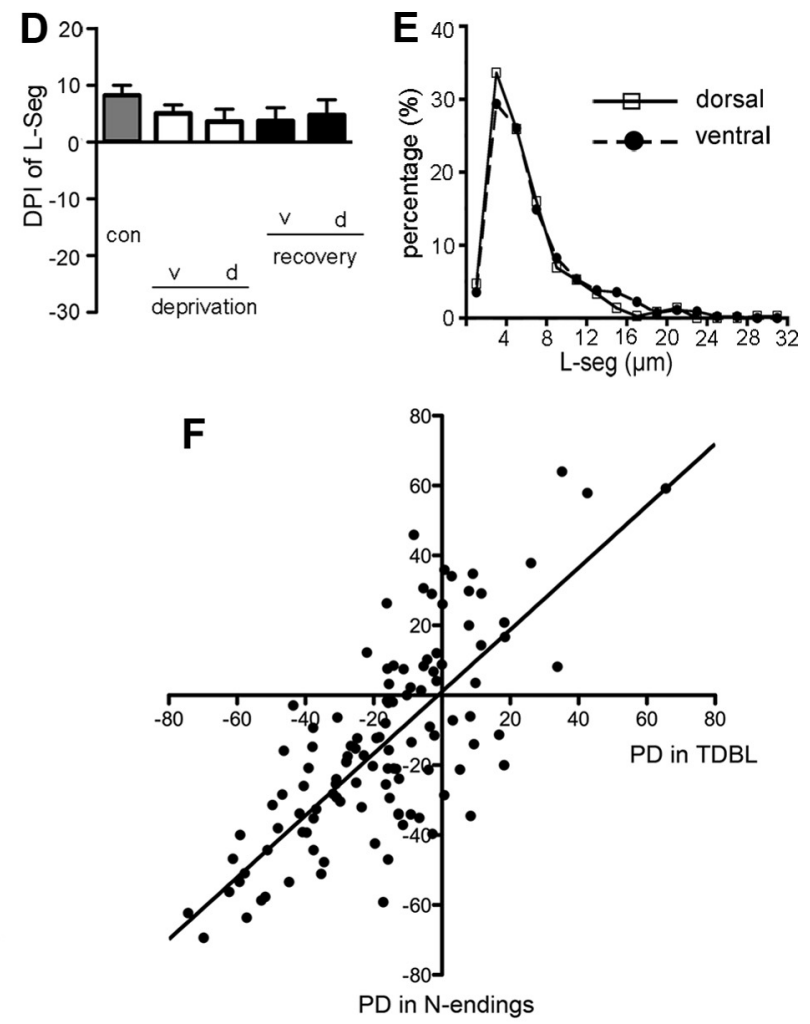

$\mathrm{PD}$ in $\mathrm{N}$-endings

Figure 5. Dendritic complexity analyses. $\boldsymbol{A}-\boldsymbol{D}$, Changes in DPl of N-endings $(\boldsymbol{A})$, N-nodes $(\boldsymbol{B})$, and N-segs $(\boldsymbol{C}$, as well as $\mathrm{L}$-seg $(\boldsymbol{D})$. Each neuron is presented as a single data point for these analyses. Neurons from animals that received XDCT transection, unilateral cochlea

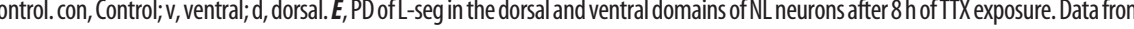
between the dorsal and ventral domains. $F$, Correlations of changes in $\mathrm{N}$-endings, with changes in TDBL after manipulations. Each neuron is presented as a single data point. Solid line indicates the linear regression $\left(r^{2}=0.58, p<0.0001\right)$. Neurons with larger PDs in TDBL tend

tent with the compartmentalization of electrical, calcium, and other biochemical signaling with single branch specificity (Rinzel and Rall, 1974; Rinzel, 1975; Sjöström et al., 2008; Branco and Häusser, 2010; Siegel and Lohmann, 2012). Similar regulatory patterns have been reported for dendritic growth induced by sensory stimulation and retraction after inactivation of glutamate receptors in tectal neurons (Rajan and Cline, 1998; Rajan et al., 1999; Sin et al., 2002).

It is also important to note that structural changes of individual branches are not determined solely by the local environment, independent of other regions of the same dendritic tree or the neuron. The earliest detectable changes of NL dendrites at the ultrastructure level after XDCT transection are cytoskeleton reductions in the basal portion of each deprived dendritic tree and a significant increase in the soma size (Deitch and Rubel, 1989a,b), indicating involvement of the whole dendritic tree and the neuron in afferent regulation of postsynaptic dendrites. This involvement is further supported by the importance of competitive inputs to dendritic maintenance (Katz and Constantine-Paton, 1988; Kossel et al., 1995; Tailby and Metha, 2004; Sorensen and Rubel, 2011). Although how a neuron integrates inputs onto different dendritic branches and then reorganizes dendritic arborization in a branchspecific manner remains unknown, computation at both global and local levels must be involved. 

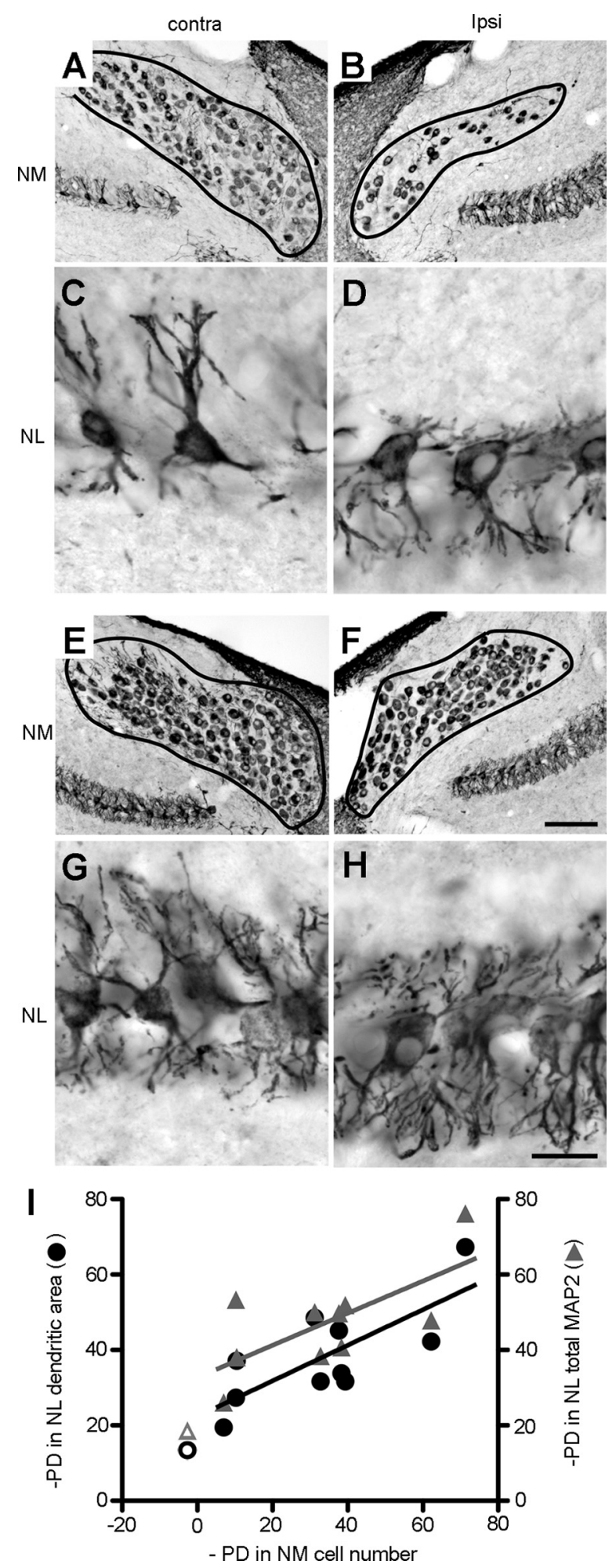

Figure 6. Correlation of cell survival in NM with dendritic maintenance in NL at $4 \mathrm{~d}$ after unilateral cochlea removal. $\boldsymbol{A}-\boldsymbol{H}$, Photomicrographs of coronal sections labeled with MAP2 immunoreactivity through NM $(\boldsymbol{A}, \boldsymbol{B}, \boldsymbol{E}$, and $\boldsymbol{F})$ and NL $(\boldsymbol{C}, \boldsymbol{D}, \boldsymbol{G}$, and $\boldsymbol{H}) . \boldsymbol{A}-\boldsymbol{D}$ were taken from one animal and $\boldsymbol{E}-\boldsymbol{H}$ from a second animal. Left $(\boldsymbol{A}, \boldsymbol{C}, \boldsymbol{E}, \boldsymbol{G})$ and right $(\boldsymbol{B}, \boldsymbol{D}, \boldsymbol{F}, \boldsymbol{H})$ columns are from the contralateral and ipsilateral sides, respectively. In both cases, the ipsilateral NM has a notable reduction in cell number compared with the contralateral NM of the same case. Within $\mathrm{NL}$, the ventral dendrites of the contralateral side and the dorsal dendrites of the ipsilateral side show clear retraction compared with the other domain of the same side. The difference between the two cases is that the first case $(\boldsymbol{A}-\boldsymbol{D})$ exhibits a much larger degree of cell death in the ipsilateral NM (72\%) compared with the second case $(\boldsymbol{E}-\boldsymbol{H} ; 11 \%)$. Correlated to this difference in transneuronal NM cell death, dendritic retractions in NL are more dramatic in the first case
At survival times longer than $48 \mathrm{~h}$ when irreversible changes in presynaptic neurons in NM take place, it appears that cell survival in NM neurons is correlated with NL dendritic maintenance. One interesting possibility is that the maintenance of remaining dendrites is affected by the integrity of NM neurons that continue to innervate these dendrites. The key players may be associated with the number and/or pattern of "silent" presynaptic appositions, such as spontaneous episodic release of transmitter (minis) and/or other trophic factors and diffusable cues independent of neuronal activity (Urbanska et al., 2008). This possibility suggests distinct mechanisms involved in short- and long-term dendritic maintenance, consistent with dramatically different retraction rates of NL dendrites after XDCT transection at the first 8-12 h compared with later time points (Deitch and Rubel, 1984). In addition, the short-term changes appear characterized by a rapid decrease in the density of the dendritic cytoskeleton (microtubule and neurofilament), whereas the late phase exhibits no additional changes in microtubule density (Deitch and Rubel, 1989a). Alternatively, cell survival in NM and long-term dendritic maintenance in NL may be influenced by similar mechanisms that depend on action-potential-mediated signals. Approaches that produce long-term changes in afferent activity without causing degeneration of presynaptic terminals would help clarify this important issue.

In summary, the rapidity, reversibility, compartmental specificity of dendritic dynamics of chicken NL neurons in response to manipulations of afferent inputs provides an advantageous model for exploring local and global cellular events governing dendritic structure. The detailed spatial and temporal information here provides a framework for analyses of the intracellular dynamics and regulatory pathways regulating the stability and plasticity of neuronal dendritic structure and function.

\section{References}

Abercrombie M (1946) Estimation of nuclear population from microtome sections. Anat Rec 94:239-247.

Benes FM, Parks TN, Rubel EW (1977) Rapid dendritic atrophy following deafferentation: an EM morphometric analysis. Brain Res 122:1-13.

Born DE, Rubel EW (1985) Afferent influences on brain stem auditory nuclei of the chicken: neuron number and size following cochlea removal. J Comp Neurol 231:435-445.

Born DE, Rubel EW (1988) Afferent influences on brain stem auditory nuclei of the chicken: presynaptic action potentials regulate protein synthesis in nucleus magnocellularis neurons. J Neurosci 8:901-919.

Born DE, Durham D, Rubel EW (1991) Afferent influences on brainstem auditory nuclei of the chick: nucleus magnocellularis neuronal activity following cochlea removal. Brain Res 557:37-47.

Branco T, Häusser M (2010) The single dendritic branch as a fundamental functional unit in the nervous system. Curr Opin Neurobiol 20:494-502.

Canady KS, Rubel EW (1992) Rapid and reversible astrocytic reaction to afferent activity blockade in chick cochlear nucleus. J Neurosci 12:1001-1009.

Caras ML, Brenowitz E, Rubel EW (2010) Peripheral auditory processing changes seasonally in Gambel's white-crowned sparrow. J Comp Physiol A Neuroethol Sens Neural Behav Physiol 196:581-599.

than in the second case. Solid lines in $\boldsymbol{A}, \boldsymbol{B}, \boldsymbol{E}$, and $\boldsymbol{F}$ outline the borders of NM. Images of NL in $\boldsymbol{C}$ $\boldsymbol{D}, \boldsymbol{G}$, and $\boldsymbol{H}$ are rotated such that dorsal is up and ventral is down. Caudorostral level of these photomicrographs are $\sim 60 \%$ in NM and $50 \%$ in NL along the caudal-to-rostral axis, corresponding to a characterized frequency of $2 \mathrm{kHz}$. I, PDs in dendritic area (black circle) and total MAP2 immunoreactivity (gray triangle) of the contralateral NL are plotted as a function of PDs in neuronal cell number between the ipsilateral and contralateral NM. Each data point represents an individual animal. Open symbols are the average from control animals. Cases with larger PDs in NM cell number tend to have larger PDs in dendritic area and total MAP2 staining in NL. Image contrast, gamma, and brightness adjustments were made in Adobe Photoshop. Scale bars: (in F) $\boldsymbol{A}, \boldsymbol{B}, \boldsymbol{E}, \boldsymbol{F}, 100 \mu \mathrm{m}$; (in $\boldsymbol{H}) \mathbf{C}, \boldsymbol{D}, \boldsymbol{G}, \boldsymbol{H}, 20 \mu \mathrm{m}$. contra, Contralateral; ipsi, ipsilateral. 
Carr CE, Konishi M (1990) A circuit for detection of interaural time differences in the brain stem of the barn owl. J Neurosci 10:3227-3246.

Cline HT (2001) Dendritic arbor development and synaptogenesis. Curr Opin Neurobiol 11:118-126.

Connors BW, Regehr WG (1996) Neuronal firing: does function follow form? Curr Biol 6:1560-1562.

Deitch JS, Rubel EW (1984) Afferent influences on brain stem auditory nuclei of the chicken: time course and specificity of dendritic atrophy following deafferentation. J Comp Neurol 229:66-79.

Deitch JS, Rubel EW (1989a) Rapid changes in ultrastructure during deafferentation-induced dendritic atrophy. J Comp Neurol 281:234-258.

Deitch JS, Rubel EW (1989b) Changes in neuronal cell bodies in N. laminaris during deafferentation-induced dendritic atrophy. J Comp Neurol 281:259-268.

Durham D, Rubel EW (1985) Afferent influences on brain stem auditory nuclei of the chicken: changes in succinate dehydrogenase activity following cochlea removal. J Comp Neurol 231:446-456.

Faherty CJ, Kerley D, Smeyne RJ (2003) A Golgi-Cox morphological analysis of neuronal changes induced by environmental enrichment. Brain Res Dev Brain Res 141:55-61.

Groc L, Petanjek Z, Gustafsson B, Ben-Ari Y, Hanse E, Khazipov R (2002) In vivo blockade of neural activity alters dendritic development of neonatal CA1 pyramidal cells. Eur J Neurosci 16:1931-1938.

Hashimoto K, Ichikawa R, Kitamura K, Watanabe M, Kano M (2009) Translocation of a "winner" climbing fiber to the Purkinje cell dendrite and subsequent elimination of "losers" from the soma in developing cerebellum. Neuron 63:106-118.

Hata Y, Tsumoto T, Stryker MP (1999) Selective pruning of more active afferents when cat visual cortex is pharmacologically inhibited. Neuron 22:375-381.

Heiman MG, Shaham S (2010) Twigs into branches: how a filopodium becomes a dendrite. Curr Opin Neurobiol 20:86-91.

Hua JY, Smear MC, Baier H, Smith SJ (2005) Regulation of axon growth in vivo by activity-based competition. Nature 434:1022-1026.

Katz LC, Constantine-Paton M (1988) Relationships between segregated afferents and postsynaptic neurones in the optic tectum of three-eyed frogs. J Neurosci 8:3160-3180.

Kleim JA, Swain RA, Czerlanis CM, Kelly JL, Pipitone MA, Greenough WT (1997) Learning-dependent dendritic hypertrophy of cerebellar stellate cells: plasticity of local circuit neurons. Neurobiol Learn Mem 67:29-33.

Kossel A, Löwel S, Bolz J (1995) Relationships between dendritic fields and functional architecture in striate cortex of normal and visually deprived cats. J Neurosci 15:3913-3926.

Lippe WR (1994) Rhythmic spontaneous activity in the developing avian auditory system. J Neurosci 14:1486-1495.

Lohmann C, Wong RO (2005) Regulation of dendritic growth and plasticity by local and global calcium dynamics. Cell Calcium 37:403-409.

Mattila PK, Lappalainen P (2008) Filopodia: molecular architecture and cellular functions. Nat Rev Mol Cell Biol 9:446-454.

Mizrahi A, Katz LC (2003) Dendritic stability in the adult olfactory bulb. Nat Neurosci 6:1201-1207.

Nagendran T, Hardy LR (2011) Calcium/calmodulin-dependent protein kinase IV mediates distinct features of basal and activity-dependent dendrite complexity. Neuroscience 199:548-562.

Parks TN, Rubel EW (1975) Organization and development of brain stem auditory nuclei of the chicken: organization of projections from n. magnocellularis to n. laminaris. J Comp Neurol 164:435-448.

Parrish JZ, Emoto K, Kim MD, Jan YN (2007) Mechanisms that regulate establishment, maintenance, and remodeling of dendritic fields. Annu Rev Neurosci 30:399-423.

Purves D, Hume RI (1981) The relation of postsynaptic geometry to the number of presynaptic axons that innervate autonomic ganglion cells. J Neurosci 1:441-452.

Rajan I, Cline HT (1998) Glutamate receptor activity is required for normal development of tectal cell dendrites in vivo. J Neurosci 18:7836-7846.
Rajan I, Witte S, Cline HT (1999) NMDA receptor activity stabilizes presynaptic retinotectal axons and postsynaptic optic tectal cell dendrites in vivo. J Neurobiol 38:357-368.

Redmond L (2008) Translating neuronal activity into dendrite elaboration: signaling to the nucleus. Neurosignals 16:194-208.

Redmond L, Ghosh A (2005) Regulation of dendritic development by calcium signaling. Cell Calcium 37:411-416.

Rinzel J (1975) Voltage transients in neuronal dendritic trees. Fed Proc 34:1350-1356

Rinzel J, Rall W (1974) Transient response in a dendritic neuron model for current injected at one branch. Biophys J 14:759-790.

Rubel EW, Fritzsch B (2002) Auditory system development: primary auditory neurons and their targets. Annu Rev Neurosci 25:51-101.

Rubel EW, Parks TN (1975) Organization and development of brain stem auditory nuclei of the chicken: tonotopic organization of n. magnocellularis and n. laminaris. J Comp Neurol 164:411-433.

Schaefer AT, Larkum ME, Sakmann B, Roth A (2003) Coincidence detection in pyramidal neurons is tuned by their dendritic branching pattern. J Neurophysiol 89:3143-3154.

Siegel F, Lohmann C (2012) Probing synaptic function in dendrites with calcium imaging. Exp Neurol. Advance online publication. Retrieved July 19, 2012. doi:10.1016/j.expneurol.2012.02.007.

Sin WC, Haas K, Ruthazer ES, Cline HT (2002) Dendrite growth increased by visual activity requires NMDA receptor and Rho GTPases. Nature 419:475-480.

Sjöström PJ, Rancz EA, Roth A, Häusser M (2008) Dendritic excitability and synaptic plasticity. Physiol Rev 88:769-840.

Smith DJ, Rubel EW (1979) Organization and development of brain stem auditory nuclei of the chicken: dendritic gradients in nucleus laminaris. J Comp Neurol 186:213-239.

Smith ZD (1981) Organization and development of brain stem auditory nuclei of the chicken: dendritic development in N. laminaris. J Comp Neurol 203:309-333.

Sorensen SA, Rubel EW (2006) The level and integrity of synaptic input regulates dendrite structure. J Neurosci 26:1539-1550.

Sorensen SA, Rubel EW (2011) Relative input strength rapidly regulates dendritic structure of chick auditory brainstem neurons. J Comp Neurol 519:2838-2851.

Stopp PE, Whitfield IC (1961) Unit responses from brain-stem nuclei in the pigeon. J Physiol 158:165-177.

Tailby C, Metha A (2004) Artificial scotoma-induced perceptual distortions are orientation dependent and short lived. Vis Neurosci 21:79-87.

Tirko NN, Ryugo DK (2012) Synaptic plasticity in the medial superior olive of hearing, deaf, and cochlear-implanted cats. J Comp Neurol 520:2202-2217.

Urbanska M, Blazejczyk M, Jaworski J (2008) Molecular basis of dendritic arborization. Acta Neurobiol Exp (Wars) 68:264-288.

Vetter P, Roth A, Häusser M (2001) Propagation of action potentials in dendrites depends on dendritic morphology. J Neurophysiol 85:926-937.

Wang Y, Rubel EW (2008) Rapid regulation of microtubule-associated protein 2 in dendrites of nucleus laminaris of the chick following deprivation of afferent activity. Neuroscience 154:381-389.

Wang Y, Cunningham DE, Tempel BL, Rubel EW (2009) Compartmentspecific regulation of plasma membrane calcium ATPase type 2 in the chick auditory brainstem. J Comp Neurol 514:624-640.

Warchol ME, Dallos P (1990) Neural coding in the chick cochlear nucleus. J Comp Physiol A 166:721-734.

Wong RO, Ghosh A (2002) Activity-dependent regulation of dendritic growth and patterning. Nat Rev Neurosci 3:803-812.

Wong WT, Faulkner-Jones BE, Sanes JR, Wong RO (2000) Rapid dendritic remodeling in the developing retina: dependence on neurotransmission and reciprocal regulation by Rac and Rho. J Neurosci 20:5024-5036.

Yuste R, Tank DW (1996) Dendritic integration in mammalian neurons, a century after Cajal. Neuron 16:701-716. 